\title{
Pair Tunneling and shot noise through a single molecule in a strong electron-phonon coupling
}

\author{
Myung-Joong Hwang, ${ }^{1}$ Mahn-Soo Choi, ${ }^{2,3, *}$ and Rosa López ${ }^{3}$ \\ ${ }^{1}$ Department of Physics, Pohang University of \\ Science and Technology, Pohang 790-784, Korea \\ ${ }^{2}$ Department of Physics, Korea University, Seoul 136-713, Korea \\ ${ }^{3}$ Department de Física, Universitat de les Illes Balears, \\ E-07122 Palma de Mallorca, Spain
}

\begin{abstract}
We investigate the electronic transport through a single molecule in a strong electron-phonon coupling regime. Based on a particle-hole transformation which is made suitable for non-equilibrium situation, we treat the pair tunneling and cotunneling on an equal footing. We propose an experimental setup to enhance the visibility of pair tunneling, which has no Franck-Condon suppression. We also discuss the shot noise characteristics.
\end{abstract}

PACS numbers: 73.63.-b, 72.10.Bg, 81.07.Nb 


\section{INTRODUCTION}

Many transport features of single molecules are ascribed to the coupling between electronic and vibrational modes $\frac{1}{2}$. Especially, the electron-phonon coupling makes the effective charging energy $U$ reduced and even negative. The negative $U$ makes the double occupancy of the molecular level energetically favorable, and allows the tunneling of pairs. Although most studies on the negative $U$ have focused on the Kondo regime ${ }^{3,4,5}$, a recent work ${ }^{6}$ pointed out that the pair tunneling can significantly affect transport above the Kondo temperature $\left(T_{K}\right)$ as well, and leads to several very unusual features ${ }^{6}$. In particular, the resonance width of the pair tunneling conductance was only determined either by the temperature (linear) or by the bias voltage (non-linear); but not by the lead-molecule hybridization as in normal sequential tunneling of single particles. Further, the pair tunneling conductance was exactly twice the normal cotunneling contribution.

However, we note that there are several physically important questions that have not been addressed yet. Firstly, there is no justification why the pair tunneling be treated separately in a sequential tunneling picture apart from the normal cotunneling processes. In this work, we use a different approach and treat both on an equal footing. Secondly, it is a general observation that the pair tunneling is usually subject to the exponential Franck-Condon suppression. Then, how can we observe the pair tunneling processes experimentally in a broad background of normal cotunneling contributions? Here we propose an experimental setup enabling one to observe the pair tunneling. Thirdly, is there a simple physical explanation why the pair tunneling conductance is exactly twice the normal cotunneling contribution? Can one attribute it to the double charge of the pair (like in a tightly bound pair objects)? We find that this is not the case.

In addition to the previous questions on average current (conductance), for a better understanding of the nature of the pair tunneling, one can also investigate the fluctuations of the current. In many systems, the effective charge of the elementary excitations has been identified by the shot noise characteristics ${ }^{8,9,10}$. Interesting questions would then be: Should the pair tunneling events give the fluctuations corresponding to the Fano factor equal to 2? How different are the pairs tunneling through the device from tightly bound pair objects? In this work, we address all these questions and provide further physical insights to the pair-tunneling transport (above $T_{K}$ ), based on particle-hole transformation. 
The paper is organized as following: In Section III we establish the particle-hole transformation, which enables us to treat the pair-tunneling processes on the same footing as the normal single-electron cotunneling processes. This transformation, augmented with the exchange of the electrode indices, is suitable for non-equilibrium (as well as equilibrium) transport. In Section III we evaluate all the relevant cotunneling rates based on the particle-hole transformation. This will make clear the difference between the underlying physical processes governing the pair tunneling and normal single electron cotunneling. Moreover, we clearly point out how the pair tunneling transport is exponentially suppressed by the Franck-Condon effect in realistic molecular devices, which was overlooked in the previous work. $\underline{\underline{6}}$ Section IV is then devoted to reproduce the results on average current (and conductance) of previous work to demonstrate the efficiency of our method. The current fluctuation noise and investigate the contribution to it from the pair tunneling processes in Section V. In Section VI a realistic experiment is proposed where one can observe the pair tunneling physics without suffering from the Franck-Condon suppression, and provide the detailed analysis of the setup. Section VII concludes the paper.

\section{PARTICLE-HOLE TRANSFORMATION}

We describe the molecular device with an Anderson-Holstein model (AHM), whose Hamiltonian has the form $\widehat{H}=\widehat{H}_{L}+\widehat{H}_{R}+\widehat{H}_{M}+\widehat{H}_{T}$. The left $(L)$ and right $(R)$ electrodes are described by the non-interacting electrons

$$
\widehat{H}_{\ell}=\sum_{k \sigma}\left(\epsilon_{k}-e V_{\ell}\right) \hat{c}_{\ell k \sigma}^{\dagger} \hat{c}_{\ell k \sigma} \quad(\ell=L, R)
$$

Here $\hat{c}_{\ell k \sigma}^{\dagger}$ creates an electron with momentum $k$ and spin $\sigma$ on the lead $\ell=\{L, R\}$. The molecule has both the electronic degrees of freedom, described by the fermion operators $\hat{d}$ and $\hat{d}_{\sigma}^{\dagger}\left(\hat{n}_{\sigma}=\hat{d}_{\sigma}^{\dagger} \hat{d}_{\sigma}, \hat{n}=\hat{n}_{\uparrow}+\hat{n}_{\downarrow}\right)$, and the vibrational mode with frequency $\omega_{0}$, described by the boson operators $\hat{a}$ and $\hat{a}^{\dagger}$ :

$$
\widehat{H}_{M}=\sum_{\sigma} \epsilon_{0} \hat{d}_{\sigma}^{\dagger} \hat{d}_{\sigma}+U_{0} \hat{n}_{\uparrow} \hat{n}_{\downarrow}-\lambda \hbar \omega_{0}(\hat{n}-1)\left(\hat{a}^{\dagger}+\hat{a}\right)+\hbar \omega_{0} \hat{a}^{\dagger} \hat{a}
$$

where $\lambda$ is the dimensionless electron-phonon coupling constant between the two degrees of freedom. The electron tunneling between the each lead and the molecule is expressed as

$$
\widehat{H}_{T}=\sum_{\ell k \sigma}\left(T_{\ell k} \hat{c}_{\ell k \sigma}^{\dagger} \hat{d}_{\sigma}+\text { h.c. }\right) \text {. }
$$


The molecule-electrode couplings are characterized by the hybridization parameters $\Gamma_{\ell} \equiv$ $2 \pi \sum_{k}\left|T_{\ell k}\right|^{2} \delta\left(E-\epsilon_{k \sigma}\right)$. As usual, we ignore the weak energy dependence of $\Gamma_{\ell}$. We also put $\Gamma=\Gamma_{L}+\Gamma_{R}$.

The molecular part $H_{M}$ is diagonalized as

$$
H_{M}=e^{+S}\left[\sum_{\sigma} \epsilon_{d} d_{\sigma}^{\dagger} d_{\sigma}+U n_{\uparrow} n_{\downarrow}\right] e^{-S}+\hbar \omega_{0} a^{\dagger} a
$$

by the canonical transformation $S=\lambda(n-1)\left(a^{\dagger}-a\right) \underline{4}, \underline{41}$ The renormalized molecular level $\epsilon_{d}$ and on-site interaction $U$ are given by $\epsilon_{d}=\epsilon_{0}+\lambda^{2} \hbar \omega_{0}$ and $U=U_{0}-2 \lambda^{2} \hbar \omega_{0}$, respectively. In the strong coupling limit $\left(\lambda^{2} \gg U_{0} / 2 \hbar \omega_{0}\right)$ the on-site interaction $U$ becomes negative so that a double occupancy of the molecular level is energetically favored to a single occupancy. Indeed, by removing the phonon degrees of freedom with a SchriefferWolf (SW) transformation, it was shown that electrons are allowed to tunnel in pairs into the molecule ${ }^{6}$; see Fig. 1 (a). However, it should be stressed that the situation considered in Ref. 6 is rather special and in fact equivalent to a negative- $U$ Anderson model (isotropic Kondo model). Earlier, it was shown in Ref. 11 that the model is, in general, equivalent to an anisotropic Kondo model (not equivalent to a negative- $U$ Anderson model).$\underline{13}$ We will come back to this issue later.

It has been proved useful to map a negative- $U$ impurity model to an equivalent model with positive interaction by a particle-hole transformation $(\mathrm{PHT}) \underline{\underline{12}}$. In the same spirit, we directly apply the PHT to the AHM keeping the phonon modes $\underline{14}$. We first choose an oneto-one correspondence $k \mapsto \tilde{k}$ such that $\epsilon_{\tilde{k}}=-\epsilon_{k}$ (we assume symmetric conduction bands). Following Refs. 11,12, we then make the PHT for down spins

$$
\hat{d}_{\downarrow} \mapsto \tilde{d}_{\downarrow}^{\dagger}, \quad \hat{c}_{L k \downarrow} \mapsto \tilde{c}_{2 \tilde{k} \downarrow}^{\dagger}, \quad \hat{c}_{R k \downarrow} \mapsto \tilde{c}_{1 \tilde{k} \downarrow}^{\dagger},
$$

keeping the up spins unchanged

$$
\hat{d}_{\uparrow} \mapsto \tilde{d}_{\uparrow}, \quad \hat{c}_{L k \uparrow} \mapsto \tilde{c}_{1 k \uparrow}, \quad \hat{c}_{R k \uparrow} \mapsto \tilde{c}_{2 k \uparrow} .
$$

It is emphasized that the lead indices for the down spins have been exchanged ( $L \rightarrow 2, R \rightarrow$ 1) compared with those for the up spins $(L \rightarrow 1, R \rightarrow 2)$. This is a small yet important difference between the mapping here and that in Refs. 11,12; the lead-index exchange is not necessary at equilibrium. Under this transformation, $H_{L}+H_{R}$ is transformed to $\widetilde{H}_{1}+\widetilde{H}_{2}$ 
with

$$
\widetilde{H}_{\ell}=\sum_{k \sigma}\left(\epsilon_{k \sigma}-e \widetilde{V}_{\ell \sigma}\right) \tilde{c}_{\ell k \sigma}^{\dagger} \tilde{c}_{\ell k \sigma}
$$

and $H_{T}$ to

$$
\widetilde{H}_{T}=\sum_{\ell k \sigma}\left(\widetilde{T}_{\ell k \sigma} \tilde{c}_{\ell k \sigma}^{\dagger} \tilde{d}_{\sigma}+\text { h.c. }\right) .
$$

The effective bias voltages $e \widetilde{V}_{\ell \sigma}$ become, in general, spin-dependent: $e \widetilde{V}_{1 \uparrow}=e V_{L}, e \widetilde{V}_{1 \downarrow}=$ $-e V_{R}, e \widetilde{V}_{2 \uparrow}=e V_{R} e \widetilde{V}_{2 \downarrow}=-e V_{L}$. For the symmetric bias, which is assumed here, they are spin-independent: $e \widetilde{V}_{1 \uparrow}=e \widetilde{V}_{1 \downarrow}=e V_{b} / 2, e \widetilde{V}_{2 \uparrow}=e \widetilde{V}_{2 \downarrow}=-e V_{b} / 2$. The tunneling amplitudes $\widetilde{T}_{\ell k \sigma}$ are also spin-dependent and given by

$$
\widetilde{T}_{1 k \uparrow}=T_{L k \uparrow}, \quad \widetilde{T}_{1 k \downarrow}=-T_{R \tilde{k} \downarrow}^{*}, \widetilde{T}_{2 k \uparrow}=T_{R k \uparrow}, \quad \widetilde{T}_{2 k \downarrow}=-T_{L \tilde{k} \downarrow}^{*} .
$$

Their spin-dependence disappears only for symmetric junctions $\left(\Gamma_{L}=\Gamma_{R}=\Gamma / 2\right): \tilde{\Gamma}_{\ell \sigma}=\Gamma / 2$ $(\ell=1,2)$. Later, we will see that the junction asymmetry affects significantly the transport properties of the device. Finally, the molecular part now takes the form

$$
e^{+\widetilde{S}}\left[\sum_{\sigma} \tilde{\epsilon}_{d \sigma} \tilde{d}_{\sigma}^{\dagger} \tilde{d}_{\sigma}+\widetilde{U} \tilde{n}_{\uparrow} \tilde{n}_{\downarrow}+a^{\dagger} a\right] e^{-\widetilde{S}}
$$

with $\widetilde{S}=\lambda\left(\tilde{n}_{\uparrow}-\tilde{n}_{\downarrow}\right)\left(a^{\dagger}-a\right)$. The effective on-site interaction $\widetilde{U} \equiv-U$ thus becomes positive, and the molecular level $\tilde{\epsilon}_{d \sigma} \equiv U / 2+\sigma \Delta_{Z} / 2$ has an effective Zeeman splitting

$$
\Delta_{Z} \equiv 2 \epsilon_{d}+U=2 \epsilon_{0}+U_{0}
$$

The eigenstates are given by $|\tilde{0}\rangle|m\rangle$ (with energy $\left.\widetilde{E}_{0, m}=m \hbar \omega_{0}\right),|\tilde{2}\rangle|m\rangle\left(\widetilde{E}_{2, m}=m \hbar \omega_{0}\right)$, and $D(\sigma \lambda)|\tilde{\sigma}\rangle|m\rangle\left(\widetilde{E}_{\sigma, m}=m \hbar \omega_{0}+\tilde{\epsilon}_{d \sigma}\right)$, where $|\tilde{0}\rangle$ (empty), $|\tilde{2}\rangle$ (doubly occupied), and $|\tilde{\sigma}\rangle$ (singly occupied by spin $\sigma$ ) are electronic states of the molecule, $|m\rangle$ is the phonon state, and $D(\alpha)=\exp \left[\alpha\left(a^{\dagger}-a\right)\right] \cdot \underline{4}, \underline{5}, \underline{11}$

In the strong coupling regime $\left(\lambda^{2} \gg U_{0} / 2 \hbar \omega_{0}\right)$, the equivalent model has $\widetilde{U} \gg \Gamma$ and $\tilde{\epsilon}_{d \sigma} \ll-\Gamma<0$; the so-called local-moment regime. The transport thus occurs only through cotunneling processes (above $T_{K}$ ). The PHT maps the electronic state $|0\rangle$ of the molecule in the original model to $|\tilde{\downarrow}\rangle \equiv \tilde{d}_{\downarrow}^{\dagger}|\tilde{0}\rangle$ in the equivalent model; likewise, $|\uparrow\rangle \mapsto|\tilde{2}\rangle,|\downarrow\rangle \mapsto|\tilde{0}\rangle$, and $|2\rangle \mapsto|\tilde{\uparrow}\rangle$. The usual cotunneling process in the original model thus corresponds to the spinpreserving cotunneling (SPC) in the equivalent model, and the pair tunneling [Fig. 2(a)] to the spin-flip cotunneling (SFC) [Fig. 2(b)]. Namely, unlike in Ref. 6, in our picture 

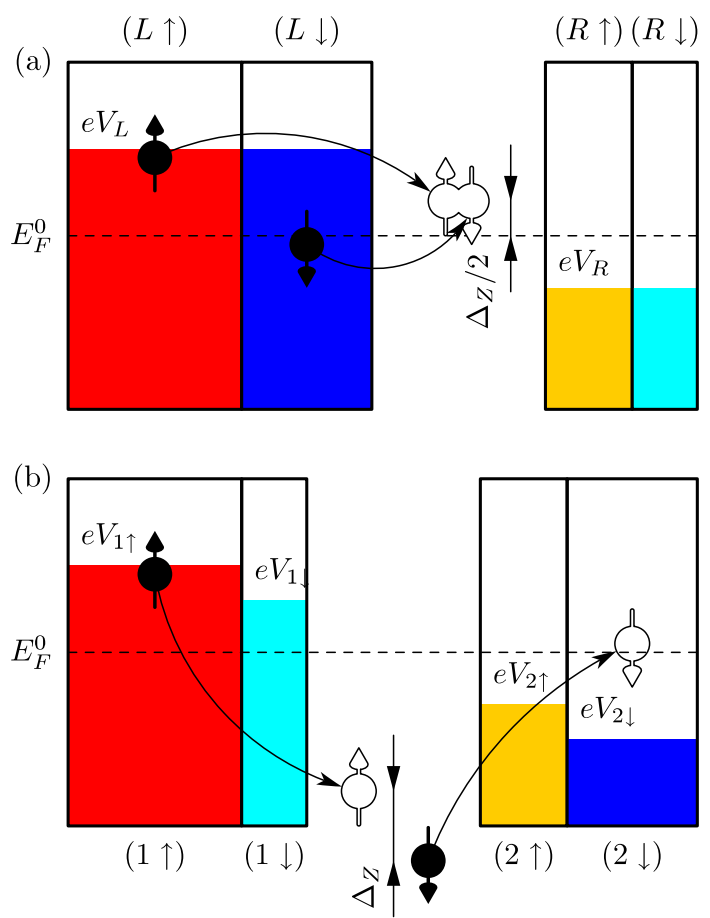

FIG. 1: (color on-line) An illustration of the particle-hole transformation. A pair tunneling in the original model (a) corresponds to a spin-flip cotunneling in the equivalent model (b). To emphasize the exchange of the electrodes in the particle-hole transformation, different widths (colors) have been used for electrode bands. Spin-preserving cotunneling processes (not shown) remain the same in both models. In (a), shown on the molecule site is the single-particle energy level for the double occupancy.

all the relevant processes are treated on an equal footing, in terms only of "cotunneling". This will allow us to infer further insight into the pair-tunneling transport based on the well-established theory of cotunneling transport ${ }^{15}$.

A few further remarks are in order: (i) In most experiments, the two leads are identical. But the mapping applies to general cases, including ferromagnetic leads $\frac{16}{}$. (ii) The transformed Hamiltonian arises from a mathematical mapping. Yet, the model itself is physical (experimentally realizable). Among others, an interesting realization will be the capacitively coupled double quantum dot (CCDQD); see Fig. 4. 
(a)

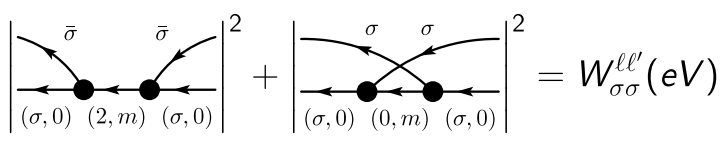

(b)

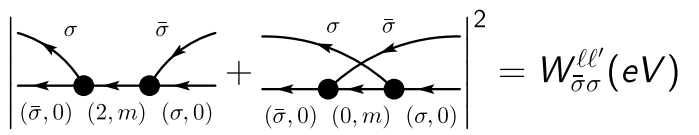

FIG. 2: Cotunneling rates in the equivalent model. (a) Spin-preserving cotunneling, corresponding to the usual single-particle cotunneling in the original model. (b) Spin-flip cotunneling, corresponding to the pair tunneling in the original model.

\section{COTUNNELING RATES}

Both the average current and the shot noise characteristics can be obtained from the rate equation

$$
\begin{aligned}
\frac{d \widetilde{P}_{\sigma}(N)}{d t}=-\sum_{\ell \ell^{\prime}, \sigma^{\prime}} \widetilde{W}_{\sigma^{\prime} \sigma}^{\ell^{\prime} \ell} \widetilde{P}_{\sigma}(N)+\sum_{\ell} \widetilde{W}_{\sigma \bar{\sigma}}^{\ell \ell} \widetilde{P}_{\bar{\sigma}}(N) & \\
& +\sum_{\sigma^{\prime}} \widetilde{W}_{\sigma \sigma^{\prime}}^{21} \widetilde{P}_{\sigma^{\prime}}(N-1)+\sum_{\sigma^{\prime}} \widetilde{W}_{\sigma \sigma^{\prime}}^{12} \widetilde{P}_{\sigma^{\prime}}(N+1) .
\end{aligned}
$$

Here $\widetilde{P}_{\sigma}(N, t)$ is the probability to find the molecule in the electronic state $|\tilde{\sigma}\rangle$ and $N$ electrons in the lead 2 at time $t$. $\widetilde{W}_{\sigma \sigma^{\prime}}^{\ell \ell^{\prime}}$ is the the rate of the cotunneling process where an electron is transferred from lead $\ell^{\prime}$ to $\ell$ and, at the same time, the molecular state changes from $\left|\tilde{\sigma}^{\prime}\right\rangle$ to $|\tilde{\sigma}\rangle$. The cotunneling rates for the spin-preserving and spin-flip processes are given respectively by

$$
\begin{gathered}
\widetilde{W}_{\sigma \sigma}^{\ell \ell^{\prime}}=\mathcal{F}_{+}^{2}(u, \lambda)\left[\frac{\tilde{\Gamma}_{\ell \bar{\sigma}} \tilde{\Gamma}_{\ell^{\prime} \bar{\sigma}}+\tilde{\Gamma}_{\ell \sigma} \tilde{\Gamma}_{\ell^{\prime} \sigma}}{2 \pi \hbar \tilde{\epsilon}_{d \sigma}^{2}}\right] F\left(e \widetilde{V}_{\ell \sigma}-e \widetilde{V}_{\ell^{\prime} \sigma}\right) \\
\widetilde{W}_{\bar{\sigma} \sigma}^{\ell \ell^{\prime}}=\mathcal{F}_{-}^{2}(u, \lambda) \tilde{\Gamma}_{\ell \sigma} \tilde{\Gamma}_{\ell^{\prime} \bar{\sigma}}\left[\frac{1}{\tilde{\epsilon}_{d \bar{\sigma}}}+\frac{1}{\tilde{\epsilon}_{d \sigma}}\right]^{2} \times \frac{F\left(\tilde{\epsilon}_{d \bar{\sigma}}-\tilde{\epsilon}_{d \sigma}+e \widetilde{V}_{\ell \sigma}-e \widetilde{V}_{\ell^{\prime} \bar{\sigma}}\right)}{2 \pi \hbar}
\end{gathered}
$$

where $u \equiv-U / 2 \hbar \omega_{0}$ and $F(E) \equiv E /\left(e^{\beta E}-1\right)$. One can immediately notice the differences between the two rates (see also Ref. 25). (i) As illustrated diagrammatically in Fig. 22, ${ }^{17}$ the SPC rate is an incoherent (classical) sum of two contributions (because the spins of the conduction electrons are different between the two corresponding diagrams) while the SFC rate is a coherent sum of two indistinguishable contributions. The latter gives rise to a constructive interference for the SFC rate, and hence $\widetilde{W}_{\bar{\sigma} \sigma}^{\ell \ell^{\prime}}$ becomes exactly twice $\widetilde{W}_{\sigma \sigma}^{\ell \ell^{\prime}}$ at 
$e V_{b} \approx 0$ and $\Delta_{Z} \approx 0$. This explains why the pair-tunneling conductance is twice the normal cotunneling contribution. (ii) They have different Franck-Condon (FC) factors

$$
\mathcal{F}_{ \pm}(u, \lambda) \equiv e^{-\lambda^{2}} \sum_{m=0}^{\infty} \frac{\left( \pm \lambda^{2}\right)^{m}}{m !} \frac{u}{m+u}
$$

The FC suppression arises at two different levels. According to the FC principle $\frac{18}{}$, each virtual tunneling amplitude itself has the $\mathrm{FC}$ suppression factor: $\left\langle\tilde{\sigma}, 0\left|H_{T}\right| \tilde{\alpha}, m\right\rangle \sim e^{-\lambda^{2} / 2}$ and $\left\langle\tilde{\alpha}, m\left|H_{T}\right| \tilde{\sigma}, 0\right\rangle \sim e^{-\lambda^{2} / 2}(\alpha=0,2)$. For the overall $\mathrm{FC}$ factor, however, the contributions from various intermediate states should be all summed up. (i) When $\hbar \omega_{0} \gg-U / 2(u \ll 1)$, $|\tilde{\alpha}, m=0\rangle$ is the only intermediate state contributing to the overall cotunneling amplitude. In this case, the overall FC factor is $e^{-\lambda^{2}}$ for both $\widetilde{W}_{\sigma \sigma}^{\ell \ell^{\prime}}$ and $\widetilde{W}_{\bar{\sigma} \sigma}^{\ell \ell^{\prime}}$. This is the limit considered in Ref. 6, corresponding to the isotropic Kondo model (negative-U Anderson model). (ii) However, if $\hbar \omega_{0} \ll-U / 2(u \gg 1)$, all the intermediate states $|\tilde{\alpha}, m\rangle$ with higher vibrational energies give finite contributions. The sum of these contributions just amounts to cancel the FC suppression factor from the individual virtual tunneling for $\widetilde{W}_{\sigma \sigma}^{\ell \ell^{\prime}}$, while it is not the case for $\widetilde{W}_{\bar{\sigma} \sigma}^{\ell \ell^{\prime}}$. Consequently, the SFC (the pair tunneling) is exponentially suppressed compared with the SPC. This is the case examined by most authors, including Refs. 11 and 4 .

We define the relative FC factor by $\gamma \equiv \mathcal{F}_{-}(u, \lambda) / \mathcal{F}_{+}(u, \lambda)$. In typical experiments with $\mathrm{C}_{60}$ molecule ${ }^{2}, U_{0} \sim 300 \mathrm{meV}$ and $\hbar \omega_{0} \sim 5 \mathrm{meV}$. Therefore, the condition for the case (i) above is hardly satisfied, and $\gamma$ can be significantly (even though not exponentially) smaller than 1, and it may be difficult to observe the pair tunneling. Below (Section VI) we will propose an experiment where the pair tunneling is not subject to the Franck-Condon suppression.

\section{CURRENT}

The average dc current is given by $I=\sum_{\sigma}\left\langle\widehat{I}_{d \sigma}\right\rangle$ with $\widehat{I}_{d \sigma}=\left(\widehat{I}_{R \sigma}-\widehat{I}_{L \sigma}\right) / 2=\left(\widetilde{I}_{2 \sigma}-\widetilde{I}_{1 \sigma}\right) / 2$, where $\widehat{I}_{\ell \sigma}=e d \widehat{N}_{\ell \sigma} / d t$ and $\widetilde{I}_{\ell \sigma}=e d \widetilde{N}_{\ell \sigma} / d t$. Referring to the PHT (Fig!1), one can identify the two contributions to the total current $I=e\left(J_{c}+J_{p}\right)$, namely,

$$
J_{c}=\left(J_{\uparrow \uparrow}+J_{\downarrow \downarrow}\right) / 2
$$

from the usual cotunneling and

$$
J_{p}=J_{\uparrow \downarrow} \widetilde{P}_{\downarrow}+J_{\downarrow \uparrow} \widetilde{P}_{\uparrow}
$$


from the pair tunneling. Here $J_{\sigma \sigma^{\prime}} \equiv \widetilde{W}_{\sigma \sigma^{\prime}}^{21}-\widetilde{W}_{\sigma \sigma^{\prime}}^{12}$, and $\widetilde{P}_{\sigma} \equiv \sum_{N} \widetilde{P}_{\sigma}(N, t=\infty)$ is the stationary probability distribution of the molecular state. Putting the cotunneling rates in Eq. (13), one can easily reproduce all the results in Ref. 6, in particular, the linear conductance (up to the FC factor $\gamma^{2}$ )

$$
G=\frac{2 e^{2} \Gamma_{L} \Gamma_{R}}{h}\left[\frac{\gamma^{2} U^{2}}{\epsilon_{d}^{2}\left(\epsilon_{d}+U\right)^{2}} \frac{\beta\left(2 \epsilon_{d}+U\right)}{2 \sinh \left[\beta\left(2 \epsilon_{d}+U\right)\right]}+\frac{1-f\left(2 \epsilon_{d}+U\right)}{\epsilon_{d}^{2}}+\frac{f\left(2 \epsilon_{d}+U\right)}{\left(\epsilon_{d}+U\right)^{2}}\right]
$$

Here we just clarify the questions raised at the beginning. In the original sequential-tunneling treatment of the pair tunneling in Ref. 6 , it is not clear why and to what extent the coherence between the subsequent pair-tunneling events can be ignored. In the present picture, the pair tunneling and normal cotunneling are treated on an equal footing, all in the cotunneling picture. Thus, in order to go beyond the sequential-tunneling treatment of the pair tunneling, viz., to the purely coherent resonant tunneling of pairs, which leads to the Kondo effect ${ }^{4}$, one has only to go to higher orders. Further, pair-tunneling contribution is exactly twice (for $\gamma=1$ ) the usual cotunneling contribution due to the interference in the SFC process (see above and Fig. 2).

\section{NOISE}

The current noise spectral density is given by (at sufficiently low frequencies $\omega \ll-U / 2$ )

$$
S(\omega)=\int_{-\infty}^{\infty} d \tau e^{+i \omega \tau}\left\langle\delta \widehat{I}_{d}(\tau) \delta \widehat{I}_{d}(0)+\delta \widehat{I}_{d}(0) \delta \widehat{I}_{d}(\tau)\right\rangle
$$

where $\delta \widehat{I}_{d} \equiv \widehat{I}_{d}-\left\langle\widehat{I}_{d}\right\rangle$. The Fano factor, defined by $S(0) / 2 e I$, is a representative characteristic of the shot noise $\left(e V_{b} \gg k_{B} T\right)$. It may reveal not only the correlated transport but also the effective charge of the carriers $\stackrel{8,9,10}{2}$. Using the quantum regression theorem $\underline{9,10,23,24}$ and the rate equation (12), we obtain current-current correlation function $\left(k_{B} T \ll e V_{b} \ll-U / 2\right.$ and $\left.\Delta_{Z} \ll-U / 2\right)$

$$
S(\omega)=2 e I-4 e^{2}\left[\frac{J_{\uparrow \downarrow}-J_{\downarrow \uparrow}}{\widetilde{W}_{p}}\right]\left[\frac{J_{\uparrow \downarrow} \widetilde{P}_{\downarrow}^{2}-J_{\downarrow \uparrow} \widetilde{P}_{\uparrow}^{2}}{1+\omega^{2} / \widetilde{W}_{p}^{2}}\right]
$$

where $\widetilde{W}_{p}=\sum_{\ell \ell^{\prime} \sigma} \widetilde{W}_{\sigma \bar{\sigma}}^{\ell \ell^{\prime}}$. The Fano factor thus has the form

$$
\frac{S(0)}{2 e I}=1-2\left[\frac{J_{\uparrow \downarrow}-J_{\downarrow \uparrow}}{\widetilde{W}_{p}}\right]\left[\frac{J_{\uparrow \downarrow} \widetilde{P}_{\downarrow}^{2}-J_{\downarrow \uparrow} \widetilde{P}_{\uparrow}^{2}}{J_{c}+J_{p}}\right]
$$

with $J_{c}$ and $J_{p}$ given by Eqs. (16) and (17). 

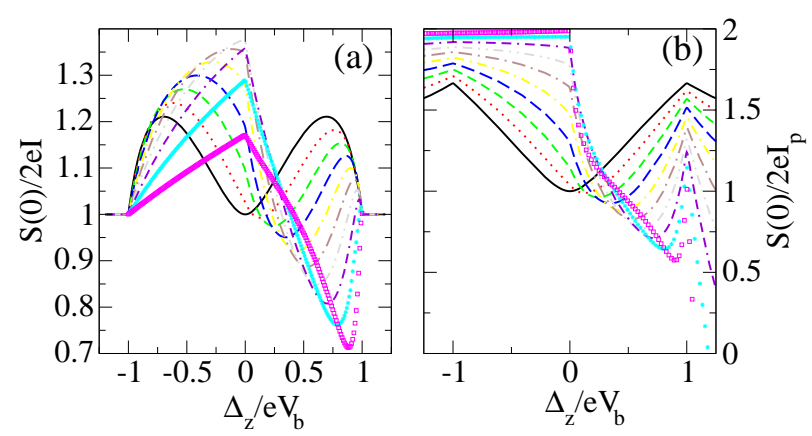

FIG. 3: (color on-line) (a) Fano factor as a function of $\Delta_{z} / e V_{b}$ for different values of $\alpha$ from 0 (black solid line) to 0.9 (violet empty square) in steps of $0.1 . \alpha \equiv\left(\Gamma_{L}-\Gamma_{R}\right) / \Gamma$ is the junction asymmetry parameter. Here $e V_{b}=500 k_{B} T$ and $\gamma=1$. (b) Fano factor with respect to $I_{p}$.

The shot noise characteristic is summarized in Fig. 3, It shows several interesting features: (i) The deviation from the Poissonian shot noise $[S(0) / 2 e I=1]$ comes entirely from the pair tunneling. This can be easily understood since the SPC in the equivalent model is an elastic process, which is known to be Poissonian $\frac{15}{}$. (ii) For the symmetric junctions $\left(\Gamma_{L}=\Gamma_{R}\right)$, the Fano factor is exactly 1 at resonance $\left(\Delta_{Z}=0\right.$ ), and increases rapidly with $\left|\Delta_{Z}\right|$ (up to $\left.\left|\Delta_{Z}\right| \sim e V_{b}\right)$. The usual sequential tunneling at resonance across symmetric junctions gives a Fano factor of $0.5^{24}$. One may be tempted to interpret it as $0.5 \times 2$ with the factor of 2 for the effective charge $2 e$ of pairs. However, this cannot be justified since the Fano factor is relative to the total current $I=e\left(J_{c}+J_{p}\right)$, but not to the pair-tunneling contribution $I_{p}=e J_{p}$. For definiteness, let us examine the pair Fano factor $S(0) / 2 e I_{p}$, as shown in Fig. 3 (b). It shows that the pair tunneling in the system cannot be interpreted as a tunneling of tightly bound pair objects. .27 Instead, we interpret it again in the cotunneling picture of the equivalent model. The SFC is an inelastic process. At $\Delta_{Z}=0$, the two channels for the inelastic cotunneling are equal, and give no additional fluctuations (the Fano factor of 1). As $\Delta_{Z}$ increases, one channel carries larger current than the other, which gives additional fluctuations $\frac{15}{}$. (iii) The shot noise turns out to be very sensitive to the junction asymmetry. Unlike single-particle sequential tunneling, the pair-tunneling even gives sub-Poissonian shot noise. This is also different from the inelastic cotunneling noise in usual QDs 15 , but this difference comes from the exchange of the lead indices in the PHT. 


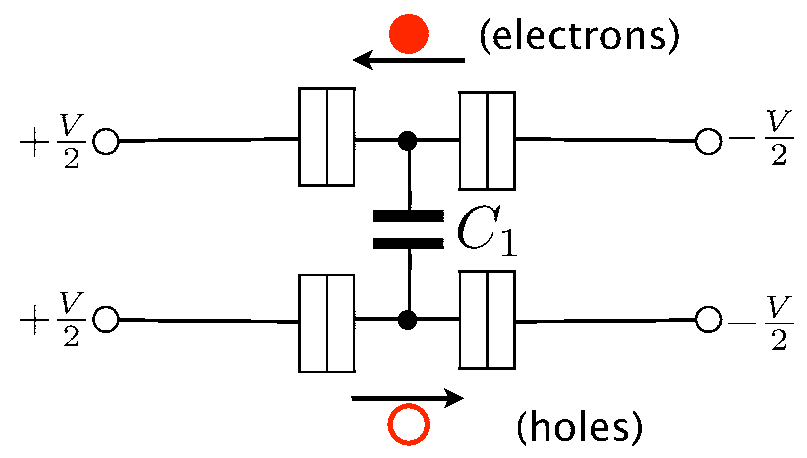

FIG. 4: (color on-line) A schematic of capacitively-coupled double quantum dots. The majority carriers through QD 1 (upper) are the electrons (red filled circle) and those through QD 2 (lower) are the holes (red empty circle). The gate voltages coupled to QDs are not shown for simplicity.

\section{TUNNELING OF ELECTRON-HOLE PAIRS}

Now we propose a experimentally feasible set-up to observe the pair tunneling transport. It consists of capacitively coupled double quantum dots, where each dot is connected to its own leads via tunneling junctions and two dots are coupled in parallel capacitively; see Fig. 4. The coupling capacitance between two dots is denoted by $C_{1}$, to be distinguished from the self-capacitance $C_{0}$ of each quantum $\operatorname{dot}(\mathrm{QD})$, which includes the junction capacitance and the gate capacitance. The tunnel junctions are specified by the hybridization parameter $\Gamma_{\ell=\{L, R\}, i=\{1,2\}}$ between QD $i$ and lead $\ell$. Since capacitance is relatively insensitive to the sample fabrication geometry (unlike exponentially sensitive hybridization), for simplicity we assume that the capacitances are symmetric over the upper and lower branches and for the left and right junctions. The gate voltages are applied oppositely to the two QDs, so that the gate-induced charges are opposite to each other:

$$
n_{g, 1}=-n_{g, 2}=n_{g}
$$

The effective carriers, say, through QD 1 are then electrons while those through QD 2 are holes, provided that the QDs are made of semiconductors ${ }^{21}$. When the QDs are ultra-small metallic grains 19,20 , the "electrons" here are the excess electrons and the holes are the deficit electrons with respect to the mean background charge.

The electrostatic energy of the double QD is given by

$$
E_{C}\left(n_{1}, n_{2}\right)=E_{1}\left(n_{1}+n_{2}-2 n_{g}\right)^{2}+E_{0}\left(n_{1}-n_{2}\right)^{2}
$$




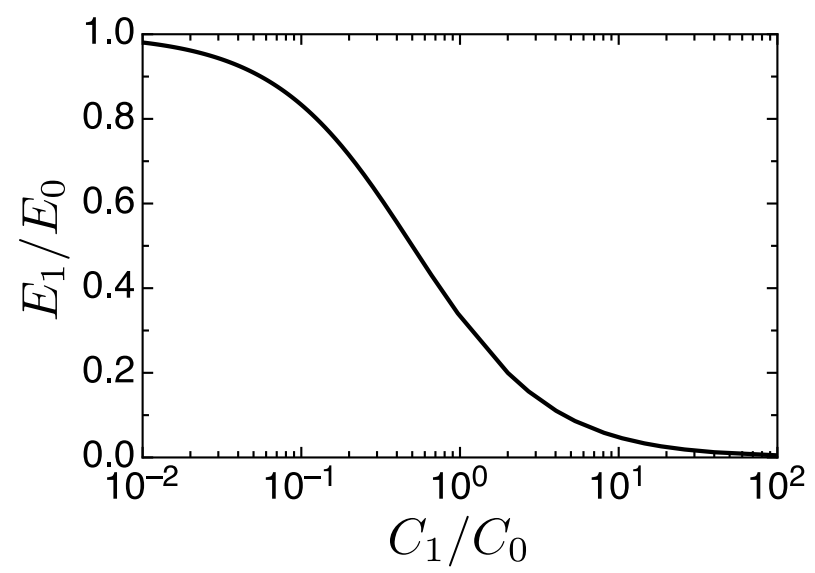

FIG. 5: The Coulomb energy ratio $E_{1} / E_{0}$ versus the capacitance ratio $C_{1} / C_{0}$. Note that for arbitrary $C_{1} / C_{0}, E_{0}$ is always bigger than $E_{1}$, leading to the negative interaction energy $U_{C}$.

where $n_{1}$ is the number of excess electrons on QD 1 while $n_{2}$ is the number of excess holes (the number of deficit electrons) on QD 2. In Eq. (23), the two Coulomb energy scales $E_{0}$ and $E_{1}$ have been defined by

$$
E_{0} \equiv \frac{e^{2}}{4 C_{0}}
$$

and

$$
E_{1} \equiv \frac{e^{2}}{8 C_{1}} \frac{1}{1+C_{0} / 2 C_{1}} .
$$

We note that the ratio $E_{1} / E_{0}$ is given by

$$
\frac{E_{1}}{E_{0}}=\frac{C_{0} / 2 C_{1}}{1+C_{0} / 2 C_{1}} \approx\left(C_{0} / 2 C_{1}\right)-\left(C_{0} / 2 C_{1}\right)^{2},
$$

and that $E_{1}$ is always smaller than $E_{0}$ for arbitrary ratio $C_{1} / C_{0}$ as illustrated ed in Fig. 5 , In particular, for $C_{1} \gg C_{0}, E_{1} / E_{0}$ becomes vanishingly small.

In this large coupling regime, Eq. (23) shows that the electrostatic energy is very low when there are same number of electrons and holes in QD1 and QD2, respectively, that is, when $n_{1}=n_{2}$. In other words, it is energetically favorable for this system to form electronhole pairs in two dots. Indeed, in the Coulomb blockade regime $\left(E_{0}, E_{1} \gg \Gamma, k_{B} T\right)$, if we set the gate voltage, so that $n_{g}=1 / 2$, then one can identify four lowest-energy charge states of the DQD, $\left|n_{1}, n_{2}\right\rangle=|0,0\rangle,|1,1\rangle,|1,0\rangle$ and $|0,1\rangle$, whose energies are given by

$$
E_{C}(0,0)=E_{C}(1,1)=E_{1}, \quad E_{C}(1,0)=E_{C}(0,1)=E_{0}
$$

Therefore, $U_{C} \equiv 2\left(E_{1}-E_{0}\right)<0$ plays the role of the negative interaction energy $U$ of the molecule and $\epsilon_{C} \equiv\left(E_{0}-E_{1}\right)>0$ is the counterpart of the energy $\epsilon_{d}$ of the singly-occupied 
states in the molecule. We emphasize that for arbitrary $C_{0} / C_{1}$, one always has $U_{C}<0$ and $\epsilon_{C}>0$; see Eq. (26) and Fig. 5. Of course, in order for the electron-hole pair state $|1,1\rangle$ (together with the empty state $|0,0\rangle$ ) to dominate the other charge states in the transport, it is required that

$$
\left|U_{C}\right|, \epsilon_{C} \gg \Gamma
$$

which in turn gives the condition

$$
\frac{C_{1}}{C_{0}} \gg \frac{1}{2\left(E_{0} / \Gamma-1\right)} .
$$

In typical experiments on small metal-grain $\mathrm{QDs}^{19}, 20,26, E_{0} / \Gamma \gtrsim 5$. It then suffices that

$$
\frac{C_{1}}{C_{0}} \gg \frac{1}{10}
$$

Large capacitive couplings have already been realized experimentally. $C_{1} / C_{0} \approx 5$ in nanoscale metallic grains ${ }^{20}$, fully satisfying the condition (30). On semiconducting quantum $\operatorname{dots}^{21} C_{1} / C_{0} \approx 0.28(0.34)$, satisfying the condition (30) only marginally and hence reducing the pair-tunneling contributions slightly from the ideal values. A very recent measurement of the cross-correlation noise through $\mathrm{CCDQD}^{22}$, even though the coupling capacitance in this experiment was rather small $\left(C_{1} / C_{0} \approx 0.1\right)$ and pair-tunneling contribution cannot be dominant, suggests that further detailed studies of the CCDQD are worthwhile.

Given the condition (30) satisfied, the calculations of the current and noise are exactly the same as in the molecular case with only one exception. The exceptional difference comes from the fact that, for example, $\Gamma_{L, 1}$ and $\Gamma_{L, 2}$ can be significantly different in realistic experiments. These junction anisotropies change, e.g., the conductance in Eq. (18) to

$$
\begin{aligned}
G=\frac{2 e^{2}}{h} \frac{\Gamma_{L, 1} \Gamma_{R, 2}+\Gamma_{L, 2} \Gamma_{R, 1}}{2} & {\left[\frac{U^{2}}{\epsilon_{d}^{2}\left(\epsilon_{d}+U\right)^{2}} \frac{\beta\left(2 \epsilon_{d}+U\right)}{2 \sinh \left[\beta\left(2 \epsilon_{d}+U\right)\right]}\right] } \\
+ & \frac{2 e^{2}}{h} \frac{\Gamma_{L, 1} \Gamma_{R, 1}+\Gamma_{L, 2} \Gamma_{R, 2}}{2}\left[\frac{1-f\left(2 \epsilon_{d}+U\right)}{\epsilon_{d}^{2}}+\frac{f\left(2 \epsilon_{d}+U\right)}{\left(\epsilon_{d}+U\right)^{2}}\right]
\end{aligned}
$$

Note that the FC factor $\gamma^{2}$ does not appear here, and hence for reasonably symmetric junctions with $\left(\Gamma_{L, 1} \Gamma_{R, 2}+\Gamma_{L, 2} \Gamma_{R, 1}\right) \approx\left(\Gamma_{L, 1} \Gamma_{R, 1}+\Gamma_{L, 2} \Gamma_{R, 2}\right)$ the pair-tunneling contribution can be clearly seen on top of the broad normal-cotunneling background. The junction anisotropy is expected to enhance the current fluctuations and hence the super-Poissonian nature of the noise characteristics. 


\section{CONCLUSION}

By exploiting the particle-hole transformation, we studied the pair tunneling through a single-molecule transistor on an equal footing to the normal cotunneling. We have clarified the nature of the pair tunneling by revealing new features of the shot noise characteristics as well as reinvestigating the average current. We also respected the general observation that pair tunneling is subject to stronger FC suppression than the normal cotunneling, and proposed an experimental setup to enhance a visibility of the pair tunneling.

\section{Acknowledgments}

This work was supported by the SRC program (R11-2000-071), the KRF Grants (KRF2005-070-C00055 and KRF-2006-312-C00543), the Second BK21, the Grant FIS2005 02796 (MEC), and the "Ramón y Cajal" program. We thank F. von Oppen for sending his presentation slides and preprint. M.-J.H thanks H.-W. Lee for helpful discussions.

* Electronic address: choims@korea.ac.kr

1 G. Cuniberti, G. Fagas, and K. Richter, Eds., Introducing Molecular Electronics (Springer, Berlin, 2005).

2 H. Park, J. Park, A. K. L. Lim, E. H. Anderson, A. P. Alivisatos, and P. L. McEuen, Nature 407, 57 (2000).

3 A.S. Alexandrov, A.M. Bratkovsky, and R.S. Williams, Phys. Rev. B 67, 075301 (2003); A.S. Alexandrov and A.M. Bratkovsky, Phys. Rev. B 67, 235312 (2003); L. Arrachea and M.J. Rozenberg, Phys. Rev. B 72, 042402 (2005); J. Mravlje, A. Rams̆ak and T. Rejec, Phys. Rev. B 72, 121403(R) (2005).

4 P. S. Cornaglia, H. Ness, and D. R. Grempel, Phys. Rev. Lett. 93, 147201 (2004).

5 P. S. Cornaglia and D. R. Grempel, Phys. Rev. B 71, 245326 (2005).

6 J. Koch, M. E. Raikh, and F. von Oppen, Phys. Rev. Lett. 96, 056803 (2006).

7 M. Büttiker, Phys. Rev. B 33, 3020 (1986); M. Büttiker, IBM J. Res. Develop. 32, 63 (1988).

8 Y. M. Blanter and M. Büttiker, Phys. Rep. 336, 1 (2000).

9 M.-S. Choi, F. Plastina, and R. Fazio, Phys. Rev. B 67, 045105 (2003). 
10 M.-S. Choi, F. Plastina, and R. Fazio, Phys. Rev. Lett. 87, 116601 (2001).

11 H.-B. Schüttler and A. J. Fedro, Phys. Rev. B 38, 9063 (1988); I. G. Lang and Y. A. Firsov, Zh. Eksp. Teor. Fiz. 43, 1843 (1962) [Sov. Phys. JETP 16, 1301 (1963)].

12 A. Taraphder and P. Coleman, Phys. Rev. Lett. 66, 2814 (1991); V. J. Emery, Phys. Rev. B 14, 2989 (1976); G. Iche and A. Zawadowski, Solid State Commun. 10, 1001 (1972); H. Shiba, Prog. Theor. Phys. 48, 2171 (1972).

13 The associated Kondo effect is out of the scope of this work.

14 One could go further to remove the phonons by the SW transformation and get the anisotropic Kondo model as in 11 . While finishing this work, we learned that Koch et al. (preprint) use the same mapping (with $\gamma=1$ ) in a related work.

15 E. V. Sukhorukov, G. Burkard, and D. Loss, Phys. Rev. B 63, 125315 (2001).

16 R. López and M.-S. Choi, in preparation (unpublished).

17 A. C. Hewson, The Kondo Problem to Heavy Fermions (Cambridge University Press, Cambridge, 1993).

18 J. Franck, Transactions of the Faraday Society 21, 536 (1926); E. Condon, Phys. Rev. 28, 1182 (1926).

19 P. Delsing, D. B. Haviland, and P. Davidsson, Czech. J. Phys. 46, 2359 (1996); H. Shimada and P. Delsing, Phys. Rev. Lett. 85, 3253 (2000); M.-S. Choi, M. Y. Choi, T. Choi, and S.-I. Lee, Phys. Rev. Lett. 81, 4240 (1998).

20 M. Matters, J. J. Versluys, and J. E. Mooij, Phys. Rev. Lett. 78, 2469 (1997).

21 I. H. Chan, R. M. Westervelt, K. D. Maranowski, and A. C. Gossard, Appl. Phys. Lett. 80, 1818 (2002); I. Chan, P. Fallahi, R. Westervelt, K. Maranowski, and A. Gossard, Physica E 17, $584(2003)$.

22 D. T. McClure, L. DiCarlo, Y. Zhang, H.-A. Engel, C. M. Marcus, M. P. Hanson, and A. C. Gossard, Phys. Rev. Lett. 98, 056801 (2007).

23 C. W. Gardiner and P. Zoller, Quantum Noise (Springer-Verlag, Berlin, 2000), 2nd ed.

24 S. Hershfield, J. H. Davies, P. Hyldgaard, C. J. Stanton, and J. W. Wilkins, Phys. Rev. B 47, 1967 (1993); A. N. Korotkov, Phys. Rev. B 49, 10381 (1994); R. Aguado and T. Brandes, Phys. Rev. Lett. 92, 206601 (2004).

25 J. Lehmann and D. Loss, Phys. Rev. B 73, 045328 (2006).

26 L. L. Sohn, L. P. Kouwenhoven, and G. Schön, Eds., Mesoscopic Electron Transport (Kluwer 
Academic Publishers, Dordrecht, 1997).

27 In Fig. 3, the noise in the region $\left|\Delta_{Z}\right| \gg e V_{b}$ is governed by thermal-assisted tunneling, and is not "shot" noise. 